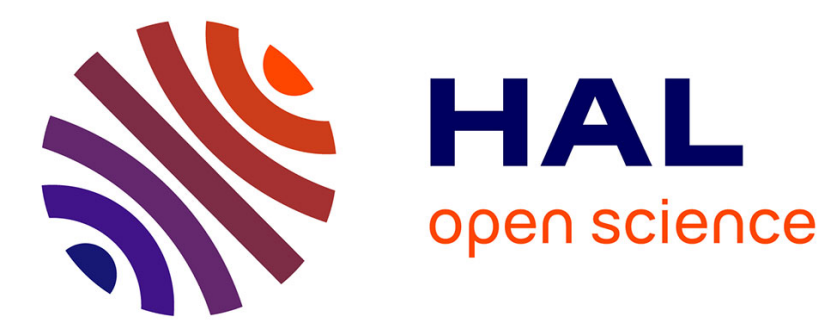

\title{
Energy efficient cluster-based routing in wireless sensor networks
}

Houda Zeghilet, Nadjib Badache, Moufida Maimour

\section{To cite this version:}

Houda Zeghilet, Nadjib Badache, Moufida Maimour. Energy efficient cluster-based routing in wireless sensor networks. 14th IEEE Symposium on Computers and Communications, ISCC'09, Jul 2009, Sousse, Tunisia. pp.CDROM. hal-00404877

\section{HAL Id: hal-00404877 https://hal.science/hal-00404877}

Submitted on 18 Jul 2009

HAL is a multi-disciplinary open access archive for the deposit and dissemination of scientific research documents, whether they are published or not. The documents may come from teaching and research institutions in France or abroad, or from public or private research centers.
L'archive ouverte pluridisciplinaire HAL, est destinée au dépôt et à la diffusion de documents scientifiques de niveau recherche, publiés ou non, émanant des établissements d'enseignement et de recherche français ou étrangers, des laboratoires publics ou privés. 


\section{Energy Efficient Cluster-based Routing in Wireless Sensor Networks}

\author{
Houda Zeghilet \\ DTISI Laboratory \\ CERIST, Algeria \\ Email: hzeghilet@mail.cerist.dz
}

\author{
Nadjib Badache \\ LSI Laboratory \\ USTHB University, Algeria \\ Email: badache@mail.cerist.dz
}

\author{
Moufida Maimour \\ CRAN laboratory \\ Nancy University, CNRS, France \\ Email:mmaimour@uhp-nancy.fr
}

\begin{abstract}
Because of the lack of a global naming scheme, routing protocols in sensor networks usually use flooding to select paths and deliver data. This process although simple and effective, is very costly in terms of energy consumption, an important design issue in sensor networks routing protocols. Cluster-based routing is one solution to save energy. In this paper, we propose a combination of an improved clustering algorithm and directed diffusion, a well-known data-centric routing paradigm in sensor networks. Our aim is to prolong the network lifetime by modifying passive clustering rules for building/maintaining the topology so an energy load balancing is achieved among the network nodes. We performed extensive computer simulations and showed that our solution outperforms original directed diffusion as well as when it is combined to passive clustering without energy considerations.
\end{abstract}

Index Terms - Routing, passive clustering, energy efficiency, Energy level, network lifetime.

\section{INTRODUCTION}

Global naming schemes (like IP addresses) are not very efficient for wireless sensor networks (WSN) due to their application requirements and the large number of deployed nodes. In most scenarios, the data associated with a sensor node is more important than the sensor itself. Because of this lack of a global addressing scheme, routing protocols in sensor networks rely heavily on flooding to discover routes and deliver data. Directed Diffusion (DD) [1] is a well-know data-centric routing protocol that deals with lack of global addressing using flooding. This mechanism, although simple and effective, can be quite inefficient because of large amount of redundant messages. These latters consume scarce resources such as bandwidth and particularly energy, an important design issue in WSN. Hence energy efficient routing protocols are highly required and motivated the proposition of many new routing protocols [2].

Hierarchical routing protocols (based on clustering techniques) have been proposed to achieve scalability and reduce the need for global coordination. They also allow for energy saving and thus prolong network lifetime by restricting most of the sensing, data processing and communication activities within clusters. Clustering can also provide load balancing if appropriately configured. Furthermore, it can be naturally combined with data-centric routing to make use of data aggregation techniques. Passive clustering (PC) [3] is a way to perform on-demand clustering to eliminate control messages overhead. It does not use any explicit control messages to maintain clusters. Instead, it piggybacks the control information on the outgoing data packets.

In wireless sensor networks, the PC algorithm was combined with DD in [4], [5] mainly to achieve energy efficiency. To determine a routing path, DD makes use of flooding in its different phases namely: interest propagation and exploratory data sending. Therefore, the main idea of the combination is to save energy in the flooding phases by allowing only clusterheads and gateways to take part in these phases. Ordinary nodes are only allowed to send data messages in the data sending phase. In [6], the selection of clusterheads and gateways are based on residual energy. They also propose to apply a periodic sleep and awake among cluster members which requires synchronisation among nodes.

All the previously cited works [4], [5], [6] concentrate traffic on a set of nodes performing flooding. We argue that this concentration can lead to a variance in energy consumption among sensor nodes and is able to cause rapid partition of the network. To overcome this problem, we proposed in [7] to use the energy consumed rate when selecting the flooding nodes. Although, the simulation results have shown an improvement of $39 \%$ in network lifetime when applied with DD, further improvements can be achieved. In this paper, we propose to use a more general energy heuristic, the energy level of nodes. Our changes applied on DD show that our mechanism outperforms DD and its PC combination proposed in [4] in terms of network lifetime, delay and delivery ratio.

The paper is organized as follows. Section II summarizes our proposal, the Energy Level-based Passive Clustering (ELPC). Simulation results are presented in section III before concluding.

\section{Energy Level-Based Passive Clustering}

Research on energy efficient routing is mainly focused on two aspects: minimizing energy cost per packet and balancing energy consumption in the network. In order to consider these two aspects, our solution consists in carrying out a passive clustering by considering the state of the nodes' batteries. Our aim is to achieve energy efficiency in terms of network lifetime, not only in terms of energy consumption.

In this paper, we propose to split the node's battery into levels. One can make a correspondence between different 
energy levels of a node and virtual sub-batteries it consumed sequentially. The energy level of a node can be computed using $L=N_{l} E_{c} / E_{i}$ where $E_{c}$ is the consumed amount of energy, $E_{i}$ is the initial one and $N_{l}$ is the suggested number of levels. For instance, if the number of levels is equal to 5 , a node with an initial energy of 100 Joules and consumed the half of its battery will have an energy level of 3 . We also introduce the notion of candidature to be a clusterhead or a gateway by defining the network energy level parameter. The value of this latter depends on the number of levels at the different nodes.

We use the same states as described in [3] and include node's energy level and the network energy level in the handled messages in addition to the identity, the state of the node and the addresses of the clusterheads. Additionally, we propose to modify the two passive clustering rules of building/maintaining the topology. A node is not allowed to declare itself as a clusterhead (or a gateway) if its energy level is higher than the network energy level. When a node takes the head of its group (or becomes a gateway), it adjusts the network energy level and sends the new value in the next ongoing data message. Furthermore, a clusterhead or a gateway will give up its role as soon as its energy level reaches the network energy level. It changes its state according to its local information and the timeout value. Finding a meaningful value for the network energy level is non-trivial. We initially take a value that corresponds to the half of the battery charge. This value is adjusted by subtracting 5\% each time the condition to be a cluster head is not satisfied.

The aim of doing so is to have the same energy level at all the nodes in a given time. We argue that this heuristic is more general than the consumed energy rate as it spreads an interval instead of a point. Assume that three nodes 1, 2 and 3 are contending to be a flooding node. If we use PC algorithm, node 1 will be selected for this as it has the smallest ID. However, if we use the energy level-based PC, the three nodes will be selected alternately. Consequently, energy consumption is balanced among the three nodes. When two nodes have the same energy level, then the nodes' identities are used to solve conflicts in declaring roles.

The resulting PC can be applied to any routing protocol in sensor networks as they mostly rely on flooding and particularly with DD. This not only reduces energy consumption as in [4], but increases the whole network survivability as it will be shown in the next section.

\section{Simulation Results}

We implemented our energy-level passive clustering (ELPC) using NS-2 [8] and compared it to the original directed diffusion (DD) and PCDD (DD with the passive clustering without energy consideration). We used the Two Phase Pull diffusion algorithm with its two flooding phases. The interest flooding are initiated by the sink and the data messages flooding are performed by the sources when events are detected in the network. Additional fields in the message header are added as attributes to represent the node's energy level and the network's one.

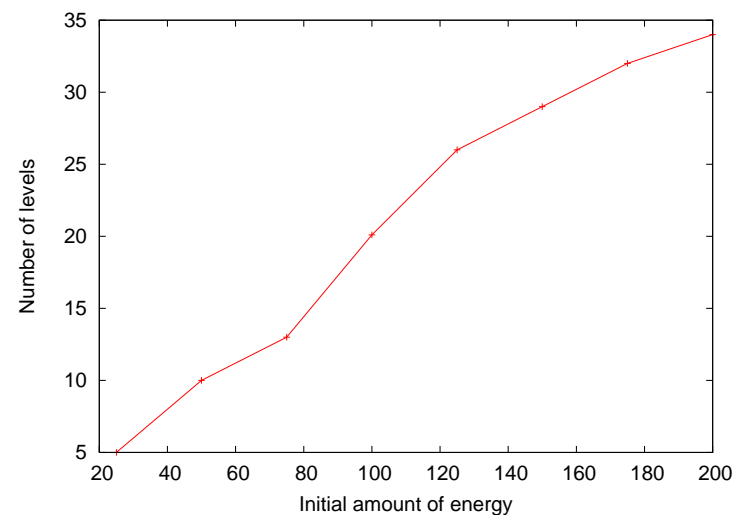

Figure 1. Number of levels corresponding to best average dissipated energy with different initial amounts of energy

For all the experiments conducted in this paper, the sensors field is of $160 \times 160 \mathrm{~m}^{2}$ unless stated otherwise. We use the IEEE 802.11 and the two-ray propagation model. The radio propagation of each sensor node reaches up to 40 meters. Each simulation runs for 1000 seconds and results are averaged over 20 randomly generated topologies.

The number of energy levels is the most important parameter to be defined. As the idea is to virtually divide the node's battery, we believe that the initial amount of energy has a great effect on this parameter. To evaluate this, we perform simulations for 100 sensor nodes where the initial energy is varied. For each amount of energy, we also vary the number of levels and record the average dissipated energy in each simulation. Figure 1 plots the number of levels corresponding to the smallest value of the average dissipated energy as a function of the initial one. It shows that the number of energy levels depends on the initial amount of energy. The average amount of energy per level can be given by $E_{m}=E_{i} / N_{l}$, then according to figure 1 , it is almost $5 \mathrm{~J}$.

To examine the effect of network topology on the different solutions, we consider both fixed zone area with variable number of nodes and fixed average density with variable zone dimensions. The traffic in both cases is the same (five sources and five sinks) unless stated otherwise. We also considered both sufficient and insufficient energy scenarios.

\section{A. Sufficient amount of energy}

In this set of simulations, nodes have sufficient amount of energy to terminate the simulation. This allows us to assess the average delay, the average dissipated energy per event and the delivery ratio. Figure 2 shows, as a function of the number of nodes, the delivery ratio of the different solutions in fixed area (a) and fixed density (b) scenarios. Passive clustering (in both PCDD and ELPC) allows for better performances in terms of delivery ratio which reaches higher values and even $100 \%$ in some cases. In fact, when increasing the nodes number or the network density, more flooded messages are observed in the network. Passive clustering allows to reduce the resulting overhead and keeps the performances of DD as it was executed 


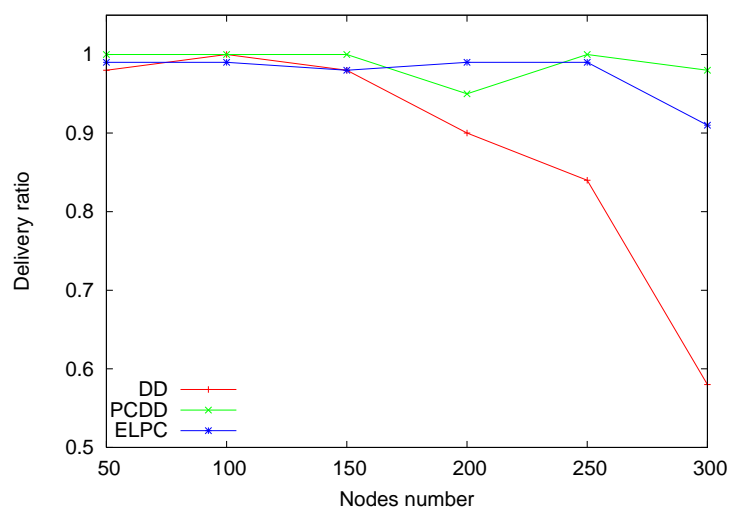

(a)

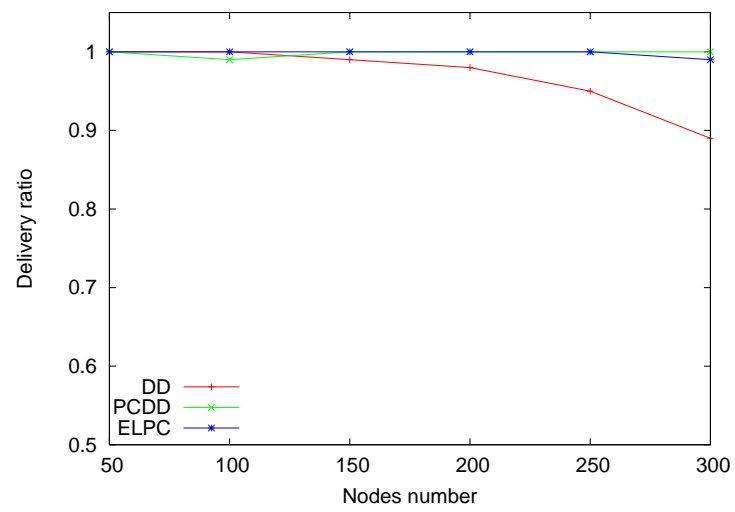

(b)

Figure 2. Delivery ratio, (a) fixed area, (b) fixed density

for small densities and networks.

\section{B. Insufficient amount of energy}

Prolonging the network lifetime is our primary goal when using energy level-based PC. We conduct these experiments with nodes assigned insufficient energy to capture network partition. We use the time to first node to die in order to see if energy consumption is fairly distributed in the network. An other metric is also used, the number of events before partition, to show the delivery capacity of the network before giving up.

a) Network topology effects: figure 3 plots the time to network partition as the network size increases. This time decreases with the number of nodes increasing. This was to be expected, as a larger number of nodes results in a higher number of floods and hence bigger amount of energy is consumed in the network. Our solution (ELPC), however, achieves better performances compared to the two others. In case of fixed area scenario (figure 3(a)), the improvement is up to $41.15 \%$ and at least $23.35 \%$ compared to PCDD and between $56.28 \%$ and $78.5 \%$ compared to the original DD. In case of fixed density (figure 3(b)), it is between $7.21 \%$ and 50\% compared to PCDD and between $55.1 \%$ and $76.17 \%$ compared DD.

The improvement is mainly due to the fact that clustering reduces the number of floods in the network and thus nodes

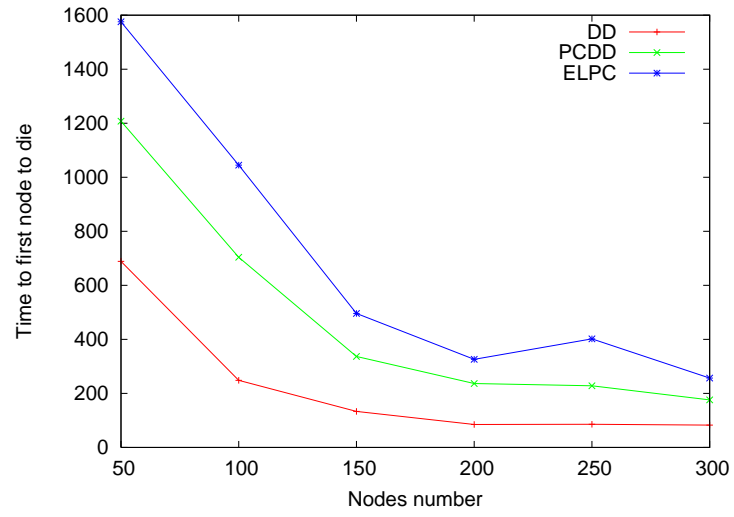

(a)

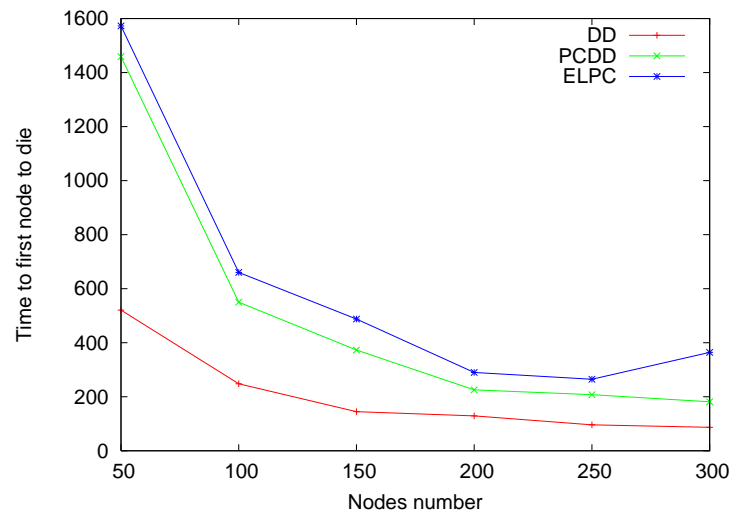

(b)

Figure 3. Network partition time (s) (a) fixed area, (b) fixed density

will consume smaller amount of energy. This helps in extending network lifetime. In our solution, an alternation of the roles is achieved. Clusterheads or gateways give up their roles when other nodes with lower energy levels are in conflict with them or when their energy level is higher than the network level. This encourages other nodes to declare themselves for these roles and, by that, leads to an increasing in the nodes lifetimes. As a consequence, the lifetime of the whole network is extended. The nodes of the network are then able to forward more data, which permits to sinks to receive a higher number of events as depicted in figure 4.

b) Traffic effects: To examine the traffic effect, we placed 100 nodes and generated traffic by a variable number of sources and sinks. When increasing network traffic, the time before network partition decreases as depicted in figure 5 . However, our solution behaves better. In the case of a single sink, the improvement is about $24 \%$ compared to PCDD and $34 \%$ compared to DD. In single source scenario, the improvement achieves $4 \%$ and $19 \%$ compared to PCDD and DD respectively. As a consequence, the number of events delivered before network partition obtained by our solution is higher than the two other solutions and by that our delivery ratio in both cases is better as shown by figure 6 . 


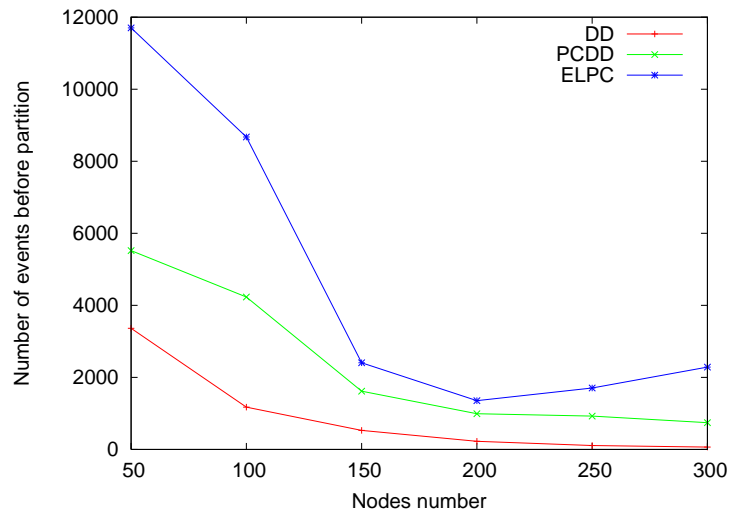

(a)

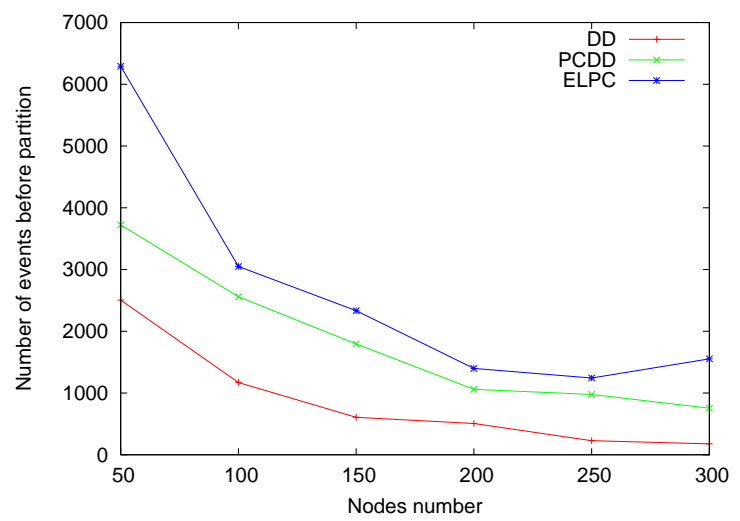

(b)

Figure 4. Number of event before partition, in (a) fixed area, (b) fixed density

\section{CONCLUSION}

In this paper, we proposed the combination of a well known routing paradigm (DD) in sensor networks with an energy efficient cluster formation algorithm. This combination gives better performances in terms of network survivability and data delivery ratio when small amounts of energy are considered. The evaluation metrics adopted in this work are of great importance in wireless senor networks as they reflect directly the network operational lifetime and its quality of service. Although, our proposal is tested on a routing protocol for sensor networks, we believe that if applied in other wireless environments (ad hoc), it will give similar performances. To validate our solution, we plan to carry out a deeper study of its different parameters.

\section{REFERENCES}

[1] C. Intanagonwiwat, et al., "Directed diffusion for wireless sensor networking," IEEE/ACM Transactions on Networking (TON), vol. 11, no. 1, pp. 2-16, 2003.

[2] K. Akkaya and M. Younis, "A survey of routing protocols in wireless sensor networks," Ad Hoc Network (Elsevier), vol. 3, no. 3, p. 325349, 2005.

[3] T. J. Kwon and M. Gerla, "Efficient flooding with passive clustering (pc) in ad hoc networks," SIGCOMM Comput. Commun. Rev., vol. 32, no. 1, pp. 44-56, 2002.

[4] V. Handziski, et al., "Improving the energy efficiency of directed diffusion using passive clustering." in EWSN, ser. Lecture Notes in Computer Science, vol. 2920, Berlin, Germany, 2004, pp. 172-187.

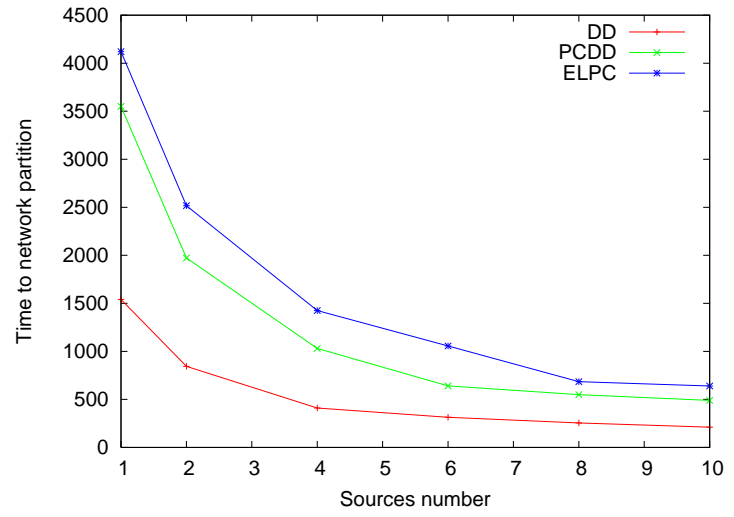

(a)

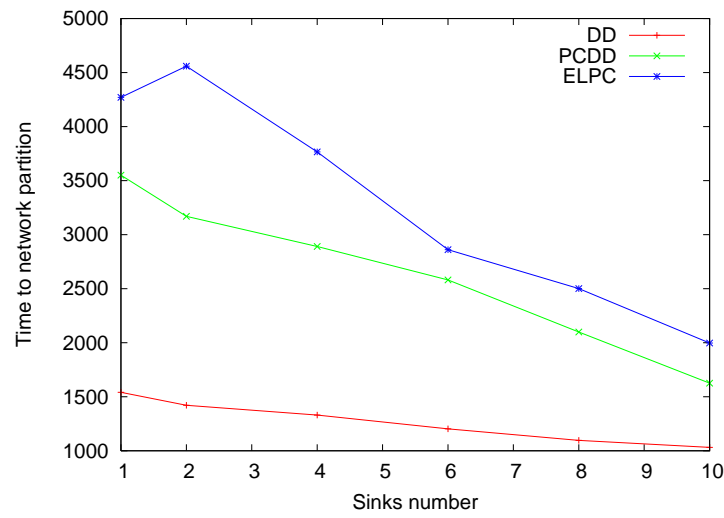

(b)

Figure 5. Network partition time (s), (a) single sink, (b) single source

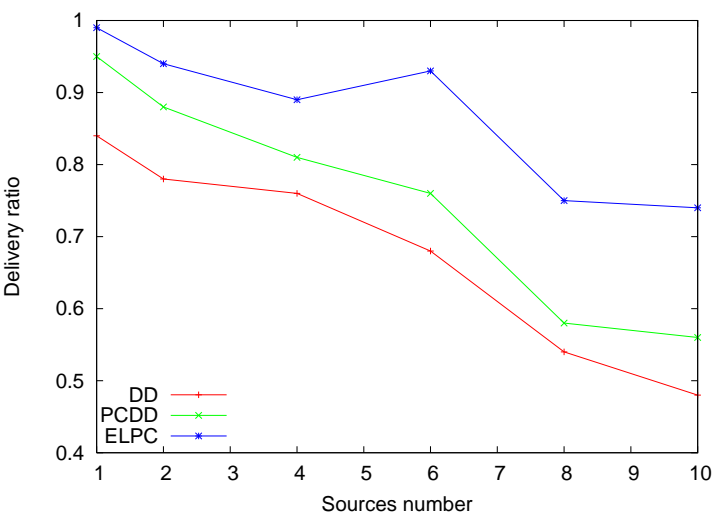

Figure 6. Delivery ratio, single sink scenario

[5] A. Rangaswamy and H. K. Pung, "Enhancement of passive cluster based routing protocol for mobile adhoc networks," in Eleventh International Conference on Computer Communications and Networks, October 2002, pp. $376-381$.

[6] M. M. or Rashid, M. M. Alam, and C. S. Hong, "Energy conserving passive clustering for efficient routing in wireless sensor network," in 9th International Conference on Advanced Communication Technology, February 2007, pp. 982-986.

[7] H. Zeghilet, "Performance improvement of passive clustering algorithm in wireless sensor networks," in Proceedings of the 4th International Conference on Networked Sensing Systems (INSS), 6-8 June 2007, pp. $53-56$.

[8] "Network simulator 2." [Online]. Available: http://www.isi.edu/nsnam/ns 